\title{
Análisis performativo de la andragogía lingüística del Inglés para el desarrollo socio-cultural
}

\section{Performative analysis of the linguistic andragogy of English for socio-cultural development}

Rocío de los Ángeles Barragán Murillo. ${ }^{1}$, Zoila Victoria Herrera Andrade. ${ }^{2}$, Esthela Isabel Colcha Guashpa. ${ }^{3} \&$ Edwin Alberto Inca Chunata. ${ }^{4}$

\begin{abstract}
.
DOI: https://doi.org/10.33262/concienciadigital.v3i2.1.1232

The article describes the result of a research process focused on the uniqueness of adults, as a group of students for foreign language proficiency, the advantages and disadvantages of intellectual maturity associated with the modern methodology of teaching the English language. This analysis will determine the importance of taking into account the peculiarities of adult students in the command of a foreign language. It starting with the basic principles of andragogy, reflecting on learning theory, and the reasons why it is consider a separate branch of pedagogy; psychological and age-related characteristics of adults that distinguish them from other groups of students are described; The interdisciplinary concept of "attention", "memory" and "thought" is studied. The entire study reveals important questions about current generations' ways of thinking and their legacy for the cultural, artistic, and social development of Latin American peoples.
\end{abstract}

Keywords: English language, Andragogy, Art and culture, Learning

${ }^{1}$ Escuela Superior Politécnica de Chimborazo, Facultad de Ciencias Pecuarias. Riobamba, Ecuador. robarragan@espoch.edu.ec

${ }^{2}$ Escuela Superior Politécnica de Chimborazo, Facultad de Recursos Naturales, Riobamba, Ecuador. zherrera@espoch.edu.ec

${ }^{3}$ Escuela Superior Politécnica de Chimborazo, Facultad Ciencias Pecuarias. Research in Teaching English for Specific purposes RITESP. Riobamba, Ecuador. ecolcha@espoch.edu.ec

${ }^{4}$ Ministerio de Educación. Educación Cultural y Artística. Quito - Ecuador, Edwin.inca@educacion.gob.ec 


\section{Resumen.}

El artículo describe el resultado de un proceso de investigación enfocado en la singularidad de los adultos, como un grupo de estudiantes para el dominio de idioma extranjero, las ventajas y las desventajas de la madurez intelectual asociadas a la metodología moderna de enseñanza del idioma inglés. Este análisis determinar la importancia de tener en cuenta las peculiaridades de los estudiantes adultos en el dominio de un idioma extranjero, iniciando por los principios básicos de la andragogía, reflexionando sobre teoría del aprendizaje, y las razones por las cuales ésta se considera una rama separada de la pedagogía; se describen características psicológicas y relacionadas con la edad de los adultos que los distinguen de otros grupos de estudiantes; se estudia el concepto interdisciplinario de "atención", "memoria" y "pensamiento". Todo el estudio revela importantes cuestionamientos sobre las formas de pensamiento generaciones actuales y su legado para desarrollo cultural, artístico y social de los pueblos Latino Americanos.

Palabras claves: Idioma Inglés, Andragogía, Arte y cultura, Aprendizaje

\section{Introducción}

El científico alemán A. Kappa, dedicó su actividad científica al estudio de la historia de la pedagogía. M. Knowles, un conocido científico en círculos pedagógicos, escribió una serie de trabajos dedicados a la solución de problemas teóricos y prácticos de la enseñanza de adultos. Knowles estaba convencido de que el profesor debería garantizar que el alumno en el proceso de aprendizaje se convierta en una persona capaz de demostrar el uso de los conocimientos adquiridos en la práctica, actuando en el marco de un sistema educativo en constante cambio. (Morales-Pacavita, 2018).

Los estudiantes deben estar preparados para el autoaprendizaje continuo, Knowles fue responsable de la formulación del tema del estudio de la andragogía que permanece en estrecha relación con la pedagogía, Mientras la andragogía presenta una serie de principios y características propias que no contradicen los principios y leyes pedagógica, sin estar contenidos en ella, la andragogía es una continuación lógica de la pedagogía. (MoralesPacavita, 2018)

\section{Metodología}

En este trabajo se utilizó un diseño de investigación experimental, para determinar las características de la epistemología o ciencia del conocimiento. Los adultos pertenecientes al grupo de experimentación fueron objeto de minuciosos test y estudios del comportamiento, cuyos resultados se exponen a continuación:

La andragogía se considera una rama separada de la pedagogía por las siguientes razones: 
- Los estudiantes regulares del sistema educativo desempeñan un papel de liderazgo en el proceso de aprendizaje, el estudiante es considerado un participante relativamente pasivo en el proceso educativo, es decir, no participa en la elección del contenido curricular de la asignatura. Es importante mencionar que el grupo de adultos participa de manera más consciente en la elección del contenido, las formas y los términos del curso de capacitación.

- Una persona adulta siempre se esfuerza por desempeñar un papel dominante en diversos procesos sociales, considerando a la filosofía, psicología, educación y autoeducación como aspectos extremadamente importantes de la vida;

- En el mundo moderno, utilizando las tecnologías de la información y las herramientas de los servicios en línea de la era de la Web 4.0, el proceso de aprendizaje se organiza de una manera nueva, en relación con la cual muchas de las nuevas responsabilidades del maestro y el alumno han modificado el proceso educativo tradicional.

- Los diferentes tipos de actividades de liderazgo son inherentes a niños y adultos, por lo que el modelo de enseñanza andragógica se basa en principios diferentes de la pedagogía que requieren una definición clara.

- Los logros teóricos y prácticos en el campo de la psicología y la fisiología demuestran que una persona puede realizar actividades educativas exitosas en cualquier etapa consciente de su vida (Fugellie, 2014).

Un adulto posee características psicológicas y específicas de la edad diferentes de otros grupos de estudiantes:

- Los adultos se reconocen a sí mismos como una persona independiente que controla el proceso de aprendizaje y percibe críticamente la función de liderazgo del maestro;

- Los adultos tienen una gran cantidad de experiencia personal, interpersonal y profesional adquirida durante un largo período de tiempo, lo que forma una visión del mundo concreta a través de la cualidad cualquier material de enseñanza pasa a través de un filtro.

- El proceso educativo debe centrarse en la importancia práctica, es decir, los adultos tienen un cierto pragmatismo en su enfoque para encontrar una solución a un problema existente;

- Los adultos están dirigidos al uso directo de conocimientos específicos adquiridos para resolver resoluciones en el contexto de la vida real;

- Las circunstancias profesionales, espacio temporal y sociales afectadas la percepción emocional especial de las actividades educativas (¿Es posible la reconstrucción de la teoría de la educación de personas adultas integrando las perspectivas humanistas, 2017).

Las características anteriores permiten darse cuenta con precisión de la singularidad de los grupos de adultos, a diferencia del grupo de estudiantes de la escuela, estudiantes de 
educación secundaria especializada y superior. Vale la pena señalar que los adultos son estudiantes con un alto grado de madurez en términos fisiológicos, psicológicos, sociales y morales. Los adultos se caracterizan por la libertad interna y la independencia financiera, que sirve como base para un comportamiento independiente y auto controlado. Por lo tanto, se puede argumentar que los estudiantes adultos son un grupo de personas exitosas que están listas y son totalmente capaces de realizar actividades educativas. La siguiente característica se deduce de esto, que consiste en el hecho de que los estudiantes adultos pueden elegir conscientemente la continuación de la educación en la edad adulta.

Las características psicológicas y fisiológicas de los adultos incluyen la autoconciencia, la autorregulación, la independencia económica, el conocimiento de los derechos y las leyes en la sociedad, la formación de un sistema de moralidad. También los separa de los grupos de estudiantes más jóvenes por la presencia de experiencia en las esferas domésticas, sociales y profesionales de la vida.

La condición del sistema de educación continua para el aprendizaje del idioma extranjero debe satisfacer las aspiraciones individuales de los adultos quienes con madurez aceptan el reto de continuar aprendiendo el idioma inglés. Se debe prestar la debida atención a conceptos interdisciplinarios como "atención", "memoria" y "pensamiento". La atención es parte fundamental en el aprendizaje que actúa como una actividad psicológica en el cual el estudiante se enfoca en un tema específico real o ideal en un momento dado (Tagle Ochoa, 2017).

Varios investigadores plantean que la atención debería tener el lado dinámico de la conciencia, la capacidad de la psique humana para concentrarse en un tema específico (Oyarce Piraud, 2008).

Existen varias clasificaciones de atención, sobre la base de las cuales es posible dividir condicionalmente el fenómeno estudiado de manera externa e interna. La atención externa está directamente relacionada con la percepción sensorial de una persona, de la realidad que lo rodea, se utiliza para conocer y transformar el material que se está estudiando. A su vez, una persona usa la atención interna para el autoestudio y la autorregulación. También se destaca la división de la atención en individual y grupal.

También se puede considerar la siguiente clasificación de atención, en relación a la experiencia obtenida del grupo experimental:

- atención involuntaria, que no depende de acciones conscientes;

- atención voluntaria, que se realiza en actividades realizadas por una persona por su propia voluntad;

- atención post-espontánea, realizada de manera arbitraria. 
La atención como categoría del método está dotada de algunas características, que incluyen:

- Distribución. - capacidad para mantener a más de un sujeto enfocado.

- Conmutación. - capacidad para cambiar el enfoque de un sujeto a otro.

- Concentración. - capacidad para enfocarse en el sujeto principal.

- Estabilidad. - capacidad para concentrarse en el sujeto principal, dando a un sujeto secundario un papel secundario a lo largo del tiempo.

En el contexto del grupo psicológico y de edad considerado en este análisis, se debe aclarar el tipo de atención a ser aplicado. Dado que el aprendizaje de adultos ocurre con mayor frecuencia en grupos, es importante resaltar la atención grupal. La atención de los adultos en el estudio de un idioma extranjero se caracteriza por ser arbitraria, debido a que los estudiantes deben aprender material útil; conmutable Después de todo, un cambio regular en los tipos de actividad del habla en un idioma extranjero hace que los adultos a menudo cambien su atención; es importante que los estudiantes aprendan a abstraer del mundo exterior para realizar actividades de aprendizaje exitosas. (Oyarce Piraud, 2008)

Además de la atención, una característica especial que debe tenerse en cuenta para tener éxito en la enseñanza de un idioma extranjero para adultos es la memoria. La memoria es un proceso mental, que incluye el procesamiento, consolidación y preservación de la experiencia adquirida en la mente humana. La memoria es una herramienta importante para activar los procesos de memorización, reproducción (extracción) y olvido de información. Por el término de retención de conocimiento, se acostumbra separar la memoria a largo plazo, a corto plazo y operativa. Existe una clasificación de la memoria, en la cual motor, figurativo, verbal - memoria lógica y emocional, según el tipo de material conservado en la memoria, se comparten la memoria cognitiva y emocional.

Una persona no puede vivir sin memoria. Dado que la memoria es una forma de reflexión mental, es responsable de las funciones de memorizar, preservar y reproducir aún más ciertos elementos de la experiencia de una persona. La memorización es la etapa básica de la memoria. Se resalta la memorización intencional (el objetivo realizado por el alumno); involuntario (realizado independientemente de la voluntad del alumno). La memorización puede tener diferentes niveles de efectividad en función de las necesidades del alumno.

El tercer factor importante que determina las características psicológicas y relacionadas con la edad de los adultos es el pensamiento. La efectividad de enseñar un idioma extranjero a adultos depende del pensamiento. El pensamiento es un proceso de actividad cognitiva, que implica una generalización e interpretación por parte de una persona de la realidad circundante. Los metodistas distinguen los siguientes tipos de pensamiento como un proceso mental de percepción de la realidad, en el que la realidad generalmente se interpreta en un sistema de interconexiones y relaciones:

- pensamiento creativo 
- pensamiento efectivo

- pensamiento lógico.

Los estudiantes adultos tienen un nivel diferente de desarrollo intelectual, en relación con el cual el pensamiento debe afectar una característica individual de cada estudiante adulto que lleva un cabo el pensamiento a través de la actividad del habla. Las actividades de aprendizaje como el análisis, la comparación y la observación son componentes del proceso de pensamiento, y el concepto, el juicio y la inferencia son formas de pensamiento.

Según el investigador (Givargizov, 1998), el pensamiento es una actividad en la persona que procesa la información recibida. Esta información se transforma en el curso de la interacción lingüística sobre la base de conceptos e ideas, sobre cualquier fenómeno almacenado en la memoria.

Por lo tanto, al enseñar a los adultos en un idioma extranjero, es necesario tener en cuenta las peculiaridades de la atención, el pensamiento y la memoria de este grupo psicológico y de edad.

En el marco de la metodología de enseñanza de lenguas extranjeras, tiene sentido considerar la andragogía lingüística, que es una sección de la metodología destinada a estudiar diversas formas de gestionar eficazmente el proceso de formación y desarrollo de la competencia comunicativa de lenguas extranjeras en estudiantes adultos. Al trabajar con estudiantes adultos, un maestro de idiomas extranjeros debe tener en cuenta los siguientes requisitos del lenguaje en el proceso de enseñanza:

- El nivel de complejidad del idioma seleccionado para el curso de idioma extranjero debe corresponder a cuán alcanzable es la meta de vida que tiene cada alumno adulto en particular.

- El significado personal del contenido seleccionado por el maestro para realizar una lección de idioma extranjero. Los adultos formulan ciertas solicitudes que satisfacen su trayectoria personal de vida, en relación con las cuales el contenido de la capacitación debe seleccionarse de acuerdo con las necesidades reales de los adultos.

- la orientación del curso para los estudiantes adultos debe estar orientado en las competencias que requerirán en situaciones de comunicación de la vida real, en otras palabras, la práctica del curso de idiomas extranjeros. Los adultos son bastante pragmáticos al recibir servicios educativos en el sistema de educación adicional, por lo que es necesario recibir de los adultos conocimientos orientados a la práctica en un volumen que exceda la parte teórica.

El Aprendizaje Cooperativo es el conjunto de procedimientos de enseñanza que parten de la organización de la clase en pequeños grupos mixtos y heterogéneos donde los estudiantes trabajan conjuntamente, de forma coordinada entre sí, para desarrollar las 4 destrezas del 
idioma (Listening, Speaking, Reading, Writing) y profundizar en su propio aprendizaje. Por tanto, requiere de la participación directa y activa del alumnado. (Azofeifa-Bolaños, 2017)

Los requisitos para la enseñanza en el campo de la educación escolar y universitaria están cambiando cualitativamente, estos cambios llevan a la necesidad de crear especiales métodos habituados a las necesidades de los estudiantes.

Como se puede observar, la mayoría de las tradiciones doxológicas y sociológicas han asumido la postura "referencial", pues han aseverado que se requiere un método específico para el aprendizaje de un grupo formado por personas adultas. Desde una perspectiva del arte y la Cultura existe una componente que aún no ha sido explotada en su máximo aporte al aprendizaje del idioma inglés, este recurso es la música, ésta es capaz de cargar significativos medios, tales como: ideas, sentimientos y rasgos de personalidad, valores sociales, creencias religiosas, e incluso mensajes ideológicos (Adorno, 1990/1941). Sin embargo, la mayoría de las veces se ha dado por sentado que el significado fundamental de la música debe buscarse en el dominio de las emociones humanas. Es decir, se considera que la música opera como un "lenguaje de los sentimientos", en el que se pueden comunicar emociones y estados de ánimo del músico a sus oyentes.

A pesar de este consenso, en la filosofía de la música y en la musicología ha persistido la controversia sobre hasta qué punto las emociones son comunicadas de manera específica por la música. Así, Davies y Kivy (Cook, 2001: 179) argumentan que la música sólo puede expresar emociones básicas tales como la alegría o la tristeza, pero no puede representar emociones más sutiles como la envidia o el orgullo pues este tipo de emociones requieren de la referencia a un objeto externo que la música no puede designar. Por el contrario, Hanslick, asegura que la música solo puede proveer una "vaga agitación", pero no logra representar emociones concretas (en Cook, 2001: 180). La teoría de la potencialidad semántica de francés afirma de manera similar que la música le presenta al oyente una vaga impresión psicológica, quien a su vez la puede interpretar (y de alguna manera reducir) mediante una etiqueta verbal (Nattiez, 1990 :126). (Cárdenas Velásquez, 2015)

El modelo de capacitación se basa en las leyes que son características de un enfoque particular para la organización del proceso de aprendizaje, presentado de forma compleja y en la forma más completa. En la práctica real, estas leyes casi nunca se implementan en su totalidad. Por lo tanto, el modelo de aprendizaje es una idea del proceso de aprendizaje, que se forma desde el punto de vista de la pedagogía.

La comparación de estos dos modelos debe comenzar con un análisis del papel de los participantes en el proceso educativo. Al modelo de entrenamiento pedagógico, papel dominante le corresponde el aprendizaje que define todos los parámetros del proceso educativo. Por razones bastante comprensibles y objetivas, como la falta de formalidad de la 
personalidad, la situación económica y social dependiente, la poca experiencia de vida, la falta de problemas graves, para cuya solución es necesario estudiar.

El aprendiz en el modelo pedagógico toma una posición dependiente y no tiene la capacidad de influir seriamente en la elección de los parámetros de entrenamiento.

En el modelo andragógico el estudiante adulto adopta una posición como miembro activo, en el proceso de aprendizaje, porque tiene una profunda necesidad de autosuficiencia, autogobierno (aunque en ciertas situaciones puede depender temporalmente de alguien). La tarea del maestro es fomentar y apoyar el desarrollo de adultos desde la dependencia total hasta el aumento del autogobierno. La característica principal del proceso de aprendizaje es el proceso de autodefinición de los parámetros de aprendizaje y la búsqueda de conocimientos, habilidades, y cualidades.

\section{CONCLUSIONES}

- El estudiante objeto del aprendizaje en el modelo pedagógico toma una posición dependiente y no tiene la capacidad de influir seriamente en la elección de los parámetros de entrenamiento.

- En el modelo andragógico el estudiante adulto adopta una posición como miembro activo, en el proceso de aprendizaje.

- La característica principal del proceso de aprendizaje es el proceso de autodefinición de los parámetros de aprendizaje y la búsqueda de conocimientos, habilidades, y cualidades.

- La cognición de la lengua extranjera permite que el individuo trascienda en sus dominios epistemológicos y la contribución en el enriquecimiento cultural y social.

- La música es una herramienta que permite abrir los horizontes del método de enseñanza aprendizaje del idioma inglés.

\section{REFERENCIAS BIBLIOGRÁFICAS.}

Es posible la reconstrucción de la teoría de la educación de personas adultas integrando las perspectivas humanistas, c. y. (2017). ¿Es posible la reconstrucción de la teoría de la educación de personas adultas integrando las perspectivas humanistas, críticas y postmodernas? Revista Electrónica Educare, 21(2); 460-485.

Azofeifa-Bolaños, J. B. (2017). Evolución conceptual e importancia de la andragogía para la optimización del alcance de los programas y proyectos académicos universitarios de desarrollo rural. Revista Electrónica Educare; , 21(1); 458-473.

Barbeitas, F. (2016). Luzes sobre a música a partir da filosofia da linguagem. Per Musi, (35); 124146. 
Barros-del Río, M. A. (2019). Cómo fomentar el espíritu crítico en los futuros docentes de inglés desde la formación: fundamentos y propuestas. Íkala, Revista de Lenguaje y Cultura, http://www.scielo.org.co/scielo.php?script=sci_arttext\&pid=S012334322019000300009\&lang=es .

Bisso, A. S. (2005). Cabildos abiertos y fiestas andinas. Dos aplicaciones del análisis performativo para el estudio de los fenómenos sociales latinoamericanos. Alteridades, 15(29); 103-124.

Bover Draganov, P. d. (2013). Andragogy in nursing: a literature review. Investigación y Educación en Enfermería; , 31(1); 86-94.

Bressiani, L. R. (2017). A utilização da Andragogia em cursos de capacitação na construção civil. Gestão \&amp; Produção, 24(4); 745-762.

Cárdenas Velásquez, D. (2015). La Compañía de Ópera Bracale en Colombia (19221933), un agente de la cultura musical del país. Historia y Sociedad; , (29); 283-312.

Cedillo, P. B.-C. (2018). MOOCEP, un método para construir cursos masivos para adultos mayores: usando una creación MOOCEP. Enfoque UTE, 9(1); 25-33.

Céspedes Guevara, J. (2010). Construcción y comunicación de significados en la música popular. CS, (5); 167-218.

Custodio, N. C.-C. (2017). Efectos de la música sobre las funciones cognitivas. Revista de NeuroPsiquiatría, 80(1); 60-69.

Díaz Larenas, C. A. (2014). Creencias de estudiantes de pedagogía en inglés sobre los modelos didácticos utilizados para la enseñanza del idioma. Núcleo, Núcleo; 26(31); 41-74.

Fugellie, D. U. (2014). Latinoamérica y el Canon. Primera conferencia de la Asociación Regional de la Sociedad Internacional de Musicología para América Latina y el Caribe (ARLAC/IMS). La Habana, 17 al 21 de marzo de 2014. Revista musical chilena, 68(222); 145-146.

Givargizov, E. Z. (1998). Patente de Estados Unidos № 5.825.122 . . Washington, DC:: Oficina de Patentes y Marcas de los Estados Unidos.

Gomes, R. C. (2012). A (etno)musicologia anglo-americana sob doze perspectivas: uma resenha do livro The new (ethno)musicologies. Per Musi; , (25); 123-126.

González R., J. P. (2001). Musicología popular en América Latina: síntesis de sus logros, problemas y desafíos. Revista musical chilena, 55(195); 38-64.

Martínez Agudo, J. d. (2013). An investigation into how EFL learners emotionally respond to teachers\&\#039; oral corrective feedback. Colombian Applied Linguistics Journal, 15(2); 265-278. 
Morales-Pacavita, O. S. (2018). Teoría andragógica: aciertos y desaciertos en la formación docente en TIC. Praxis \&amp; Saber, 9(19); 161-181.

Oyarce Piraud, M. (2008). Grammatical sensitivity: correlational-explanatory study on brain dominance and EFL training to improve gains. Colombian Applied Linguistics Journal, (10); 93-111.

Tagle Ochoa, T. D. (2017). Cómo fomentar el espíritu crítico en los futuros docentes de inglés desde la formación: fundamentos y propuestas. Folios, http://www.scielo.org.co/scielo.php?script=sci_arttext\&pid=S012348702017000100009\&lang=es . 


\section{PARA CITAR EL ARTÍCULO INDEXADO.}

Barragán Murillo, R. de los Ángeles, Herrera Andrade, Z. V., Colcha Guashpa, E. I., \& Inca Chunata, E. A. (2020). Análisis performativo de la andragogía lingüística del Inglés para el $\begin{array}{lllll}\text { desarrollo socio-cultural } \quad \text {. ConcienciaDigital, } & 3(2.1), & \text { 177-187. }\end{array}$ https://doi.org/10.33262/concienciadigital.v3i2.1.1232

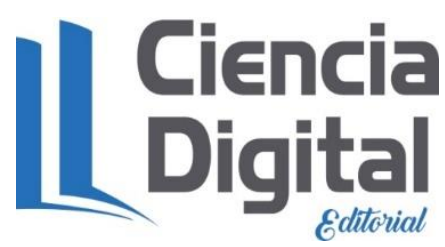

El artículo que se publica es de exclusiva responsabilidad de los autores y no necesariamente reflejan el pensamiento de la Revista Conciencia Digital.

El artículo queda en propiedad de la revista y, por tanto, su publicación parcial y/o total en otro medio tiene que ser autorizado por el director de la Revista Conciencia Digital.

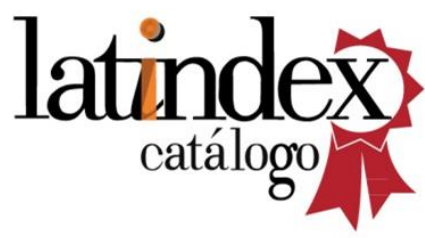

